\title{
Portugal Centre for Evidence Based Practice: An affiliate centre of the Joanna Briggs Institute - Informaçáo corrente
}

Joanna Briggs PORTUGAL CENTER FOR

Institute EVIDENCE BASED PRACTICE

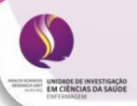

3 de março de 2009

Reunião na Unidade de Investigação em Ciências da Saúde - Enfermagem (UICISA-E), da Escola Superior de Enfermagem de Coimbra (ESEnfC) com o Prof. Dr. Alan Pearson (Diretor Executivo do Instituto Joanna Briggs - JBI) em que se discutiu a possibilidade de colaboração com o JBI.

\section{2 a 5 de fevereiro de 2010}

Curso JBI Comprehensive Systematic Review Training Program, em Thames Valley University, Londres, 6 investigadores da UICISA-E.

\section{6 de dezembro de 2010}

Seminário sobre Revisão Sistemática da Literatura (RSL) segundo a abordagem JBI no The Doctoral Programme in Health Sciences, da Faculdade de Medicina de Coimbra (FMUC)/ESENFC: Research on Social Areas.

\section{2 de dezembro de 2010}

Submissão final da candidatura a centro afiliado JBI. Assinado Memorandum of Understanding.

\section{7 de março de 2011}

UICISA-E torna-se oficialmente um Centro Afiliado do JBI, com a designação de Portugal Centre for Evidence Based Pratice: An Affiliate Centre of the Joanna Briggs Institute (PCEBP).

\section{6 de julho de 2011}

$41^{\text {a }}$ Reunião do Comité de Diretores (nódulo Europeu) via teleconferência.

\author{
Atividades Relevantes Realizadas \\ e Planeadas no Período 2009-2012
}

4 de novembro de 2011

Submetido o primeiro protocolo para RSL ao The JBI Synthesis Science Unit (SSU).

\section{7 a 12 de novembro de 2011}

JBI International Convention MISSION: IMPOSSIBLE? Evidence-based practice \& the future of global health e $42^{\circ}$ Reunião do Comité de Diretores, Adelaide Austrália.

\section{2 de janeiro de 2012}

Seminário sobre RSL segundo a abordagem JBI no The Doctoral Programme in Health Sciences, da FMUC/ESENFC: Research on Social Areas.

\section{7 de fevereiro de 2012}

Formação sobre RSL segundo a abordagem JBI numa conferência em Viseu.

\section{6 de março de 2012}

Seminário sobre RSL segundo a abordagem JBI na Unidade Curricular Dissertação do Curso de Mestrado em Enfermagem do Idoso, ESEnfC.

\section{3 de março de 2012}

Aprovado protocolo intitulado "The effectiveness of nonpharmacological nursing interventions in elderly with depressive disorders: a systematic review", disponivel em http://connect.jbiconnectplus.org/ ViewSourceFile.aspx? 06433 .

\section{3 e 24 de abril de 2012}

1st Annual Meeting of the European JBI Centre Directors, Antuérpia. 
24 de maio de 2012

$43^{\mathrm{a}}$ Reunião do Comité de Diretores (nódulo Europeu) via teleconferência.

\section{1 de junho de 2012}

Workshop sobre RSL segundo a abordagem JBI no Alexander Technological Educational Institute of Thessalonik, Grécia.

\section{2 de junho de 2012}

Workshop sobre RSL segundo a abordagem JBI no III Congresso de Investigação em Enfermagem IberoAmericano e de Países de Língua Oficial Portuguesa, na ESEnfC, Portugal.

\section{2 julho de 2012}

Integração de uma bolseira de investigação no projeto PCEBP.

\section{3 e 5 de julho de 2012}

Curso na ESEnfC sobre RSL segundo a abordagem JBI.

\section{Atividades Planeadas - 2012}

Concluir pesquisa bibliográfica para a RSL, cujo protocolo já foi aprovado.

Registar um novo título de revisão e elaborar o respetivo protocolo.

Participar na Reunião do Comité de Diretores e no 8th Biennal Joanna Briggs International Colloquium 2012 - Channeling the Rivers of Knowlegde to Improve Global Policy and Practice, de 12 a 16 de novembro de 2012, Chiang Mai, Tailândia.

Apresentar, no 8th Biennal Joanna Briggs International Colloquium 2012, o póster intitulado "The Effectiveness of Nonpharmacological Nursing Interventions in Elderly with Depressive Disorders: A Systematic Review".

Organizado por Daniela Filipa Batista Cardoso (Licenciada em Enfermagem, Bolseira de Investigação na UICISA-E no projeto Portugal Centre for Evidence Based Practice: an Affiliate Centre of the Joanna Briggs Institute) 